\title{
Generalized $t-t^{\prime}-J$ model: parameters and single-particle spectrum for electrons and holes in copper oxides.
}

\author{
V. I. Belinicher, A. L. Chernyshev, and V. A. Shubin \\ Institute of Semiconductor Physics, 630090, Novosibirsk, Russia
}

(January 20, 2018)

A microscopically based Hamiltonian of the generalized $t-t^{\prime}-J$ model is presented. Two types of the additional $t^{\prime}-$ terms are discussed. The numerical range of the amplitudes corresponding to the additional $t^{\prime}$-terms for the real $\mathrm{CuO}_{2}$ planes is derived from the three-band model calculations. Using the variational spin-polaron approach the singlecarrier dispersions in the generalized $t-t^{\prime}-J$ model are calculated both for the hole- and electron-doped systems. The hole and electron band minima are found to be at points $( \pm \pi / 2, \pm \pi / 2),(0, \pm \pi)$, and $( \pm \pi, 0)$, respectively. The band minima shifts $\left|\Delta_{(0, \pi)-(\pi / 2, \pi / 2)}\right|$ are not small $(\sim J)$. The bandwidths for both cases of doping are found to be $1.5-4.0$ times larger than those in the $t-J$ model.

75.10.Jm, 75.30.Ds

\section{INTRODUCTION}

There is a general agreement that the three-band Hubbard model is an appropriate basis for consideration of the spin and charge excitations in the $\mathrm{CuO}_{2}$ planes of high-temperature superconductors [1] 3]. One of the interesting problems for these systems is the hole energy spectrum. One-hole energy calculation in the framework of the above mentioned model has been done by Barabanov, Kuzian, Maksimov, and Uimin [4,5] who used the variational approach. This method seems to be too complicated since the characteristic energy scale of the three-band model is a few electron-volts while that of the hole energy spectrum is several tenths of an electron-volt. Therefore, it is natural to obtain first the low-energy limit of the three-band model [6 12] and then to investigate the spin and charge degrees of freedom in the framework of this effective model [13 17].

It is widely accepted by now that the simple model which contains in itself the interacting spin and charge degrees of freedom is the so called $t-J$ model

$$
H_{t-J}=t \sum_{\langle i j\rangle, \alpha} \tilde{c}_{i, \alpha}^{\dagger} \tilde{c}_{j, \alpha}+J \sum_{\langle i j\rangle} \mathbf{S}_{i} \mathbf{S}_{j}
$$

where $\langle i j\rangle$ denotes the nearest neighbor sites, $\mathbf{S}_{i}$ is the local spin operator, $\tilde{c}_{i, \alpha}^{\dagger}\left(\tilde{c}_{i, \alpha}\right)$ is the constrained fermion creation (annihilation) operator. The explicit form of this constraint depends on the type of doping.
The considerable attention that this model has received originates partly from the fact that it can be derived from the above mentioned three-band or more general $d-p$ models which take into account the detailed electronic structure of the copper oxides. Recently it has been shown [18] that this derivation can be done quantitatively for the real $\mathrm{CuO}_{2}$ planes by the use of an additional calculation of some experimentally observable values.

The behavior of a single quasiparticle in the $t$ - $J$ model has been studied intensively using both analytical and numerical techniques [14,15, 19, 20]. These investigations have clearly shown that the naive tight-binding picture is completely inadequate for the carrier motion on the antiferromagnetic background. Namely, for the realistic $t / J \simeq 2-3$ the bandwidth is of the order of $2 J$, not $W_{0}=8 t$. This strong (of the order of 10 times) bandwidth suppression results from the distortion of the spin background by the carrier hopping from one sublattice to the other. In this situation, when the characteristic energy scale becomes $J$, even small (compared to $t$ ) single-sublattice hopping $t^{\prime}$ can be the key parameter for the subtle details of the energy spectrum and other features of the doped systems. This is clear since the singlesublattice motion does not disturb the spin background. Hence, a careful analysis of the low-energy single band limit of the realistic $d-p$ models should be done keeping all essential terms over the $t^{\prime} / J$ (not $t^{\prime} / t$ ) parameter.

The simplest form of the additional $t^{\prime}$-terms can be written as

$$
H_{t^{\prime}}=t^{\prime} \sum_{\langle i j\rangle_{2}, \alpha} \tilde{c}_{i, \alpha}^{\dagger} \tilde{c}_{j, \alpha}
$$

emphasizing that the nonzero O-O hopping provides large enough transfer amplitude to the next-nearest $\left(\langle i j\rangle_{2}\right)$ neighbor 20,21. This term (2) alone has been found to be responsible for the $\mathrm{CuO}_{2}$ plane electron-hole asymmetry and to be useful for the interpretation of the recent angle-resolved photoemission experiments [22].

The goal of the present paper is to exhibit the general form of the additional $t^{\prime}$-terms which one can get from the three-band Hubbard or the other first-principle models, to determine numerical ranges of all essential parameters for the real $\mathrm{CuO}_{2}$ planes, and to calculate a single-particle dispersion using the spin-polaron ideas. Namely, we will demonstrate that two types of the additional $t^{\prime}$-terms naturally originate in the single-band model: (i) the terms arising from the O-O hopping, and (ii) the second-order high-energy channels terms [23 25]. 
The importance of both of them will be clearly shown. The first ones are the key parameters for the band minima shifts, while the second ones lead to the growing of the bandwidth. A quite similar model was investigated in the mean-field approximation for a special range of parameters in the work by Onufrieva et al [26]. This approximation neglects some essential features of the hole (electron)-spin interaction and thus has only a qualitative character.

Single-hole energy calculation in the limiting case of the three-band model using the self-consistent Born approximation has been done in the work by Starykh et al [27. In spite of neglecting some features of the problem, the results of this approach are in a qualitative agreement with the present paper.

The quantitative reduction of the three-band model developed in Ref. 18 for the real $\mathrm{CuO}_{2}$ system provides the method of the derivation of a set of the realistic ranges for all additional $t^{\prime}$-terms. We will show that the band minima shifts as well as the bandwidths are not small for these ranges of parameters. Also, the density of states (DOS) characteristic features will be discussed.

The paper is organized as follows. In Sec. II we give the general form of the corrections to the Hamiltonian (1), discuss them, and show the possible parameters ranges. In Sec. III we discuss our results for the spinpolaron dispersions and for the DOS features. Finally, in Sec. IV, we draw conclusions.

\section{REALISTIC LOW-ENERGY MODEL}

The most accurate form of the effective $t^{\prime}$-terms that follows from the three-band Hubbard model is [18,28]:

$$
\begin{aligned}
H_{t^{\prime}}= & t^{\prime} \sum_{\langle i j\rangle_{2}, \alpha} \tilde{c}_{i, \alpha}^{\dagger} \tilde{c}_{j, \alpha}+t^{\prime \prime} \sum_{\langle i j\rangle_{3}, \alpha} \tilde{c}_{i, \alpha}^{\dagger} \tilde{c}_{j, \alpha} \\
& +t^{N} \sum_{\langle i l j\rangle, \alpha} \tilde{c}_{i, \alpha}^{\dagger} \tilde{c}_{j, \alpha} \hat{N}_{l}+t^{S} \sum_{\langle i l j\rangle, \alpha \beta} \tilde{c}_{i, \alpha}^{\dagger} \boldsymbol{\sigma}_{\bar{\beta} \bar{\alpha}} \tilde{c}_{j, \beta} \mathbf{S}_{l}
\end{aligned}
$$

where $\langle i j\rangle_{2(3)}$ denotes the second (third) next-nearest neighbor sites, $\langle i l j\rangle$ denotes the three nearest neighbor sites $(\langle i l\rangle,\langle j l\rangle), \hat{N}_{l}$ is the number of fermions operator, $\mathbf{S}_{l}=\frac{1}{2} c_{l, \alpha}^{\dagger} \boldsymbol{\sigma}_{\alpha \beta} c_{l, \beta}$ is the local spin operator, $\boldsymbol{\sigma}$ is the Pauli matrix, $\bar{\alpha}=-\alpha$. The differences in the explicit form of the $\tilde{c}_{i, \alpha}^{\dagger}\left(\tilde{c}_{i, \alpha}\right)$ operators for the hole- and electron-doped systems as well as the signs of $t^{\prime}, t^{\prime \prime}, t^{N}, t^{S}$ will be discussed later. Now we will consider the origin of the $t^{\prime}, t^{\prime \prime}$ and $t^{N}, t^{S}$ terms.

Let us begin from the last ones. The second-order perturbation treatment of the usual Hubbard model near half filling provides the effective superexchange interaction (Eq. (11) and the so called 'three-site spin-dependent hopping' 24,29 31. Their importance for both the spectrum of the charge excitation and the hole-hole interaction was noted 29. These terms in Eq. (3), manifestly, have the rotationaly invariant form. In the case of the usual Hubbard model mapping to the $t-t^{\prime}-J$ model, the expressions for the $t^{N}, t^{S}$ terms have the simplest form:

$$
t^{N}=-\frac{1}{2} t^{S}=\frac{t^{2}}{2 U}=\frac{J}{8}
$$

It should be noted that this expression (4) is valid for both types of doping, and that the signs of $t^{N}$ and $t^{S}$ are the same for the electron and hole due to the second-order nature of these terms. What would one expect from the three- or more-band Hubbard model mapping?

Much more high-energy channels (triplet, etc.) are opened for the virtual second-order transitions in comparison with the usual Hubbard singlet only case [28]. This leads to two effects: (i) $t^{N} \neq-\frac{1}{2} t^{S}$, and (ii) $t_{h}^{N, S} \neq t_{e}^{N, S}$, where $e$ and $h$ denote the electron and hole hopping integrals, respectively. The exact expressions for $\left(t^{N}\right)_{e, h}$ and $\left(t^{S}\right)_{e, h}$ were derived in Refs. 12,28. In spite of the contribution of the highest states, the lowest singlet is the most important. The main features of these terms in the effective Hamiltonian (3) remain unchanged, i.e.: (i) $\operatorname{sign}\left(t^{N}\right)=-\operatorname{sign}\left(t^{S}\right)=+1$ both for the electron and hole, and (ii) $\left|t^{N}\right| \sim\left|t^{S}\right| \simeq J / 4$, so that they are not negligible.

In addition, one can point out that at the mean-field level, $\hat{N}$ and $\mathbf{S}$ operators should be replaced with their averages $\langle\hat{N}\rangle$ and $\langle\mathbf{S}\rangle$, which effectively leads to:

$$
H_{M F}^{N, S}=\tilde{t}^{\prime} \sum_{\langle i j\rangle_{2}, \alpha} \tilde{c}_{i, \alpha}^{\dagger} \tilde{c}_{j, \alpha}+\tilde{t}^{\prime \prime} \sum_{\langle i j\rangle_{3}, \alpha} \tilde{c}_{i, \alpha}^{\dagger} \tilde{c}_{j, \alpha}
$$

with $\tilde{t}^{\prime} \equiv 2 \tilde{t}^{\prime \prime}=2\left(\langle\hat{N}\rangle t^{N}+\langle\mathbf{S}\rangle t^{S}\right)$, where the additional factor 2 for the diagonal hopping integral $\tilde{t}^{\prime}$ as compared to the 'oversite' $\tilde{t}^{\prime \prime}$ arises from the two possible ways for the virtual processes on the square lattice. Thus, the second-order terms in the Hamiltonian (3) can be approximately considered as the renormalizations of the 'bare' $t^{\prime}, t^{\prime \prime}$ amplitudes (5). As was noted, these renormalizations do not change the signs under changing of the doping type.

Now, return to the first two terms of the Hamiltonian (3). They are the first-order terms arising in the firstprinciple models for the $\mathrm{CuO}_{2}$ plane from the nonzero O-O hopping. While including of the $t^{\prime}$-term is evident 15,20,21, including of the $t^{\prime \prime}$-term requires an additional explanation. Physically, only the $t^{\prime}$-term can arise for the next-nearest neighbor $\mathrm{CuO}_{4}-\mathrm{CuO}_{4}$ local states due to the $t_{p p}$ matrix element $[7,32$. However, as was shown [6, $8,28,33$ the correct state of the oxygen low-energy degrees of freedom are the ortogonalized Wannier states. Consequently, some 'unphysical' transition amplitudes to the more distant neighbors arise. These amplitudes fall rapidly with distance, and only the $t^{\prime}, t^{\prime \prime}$-terms should be kept in the low-energy model [28].

As was recently proposed in many works, the absolute sign of the $t^{\prime}$-terms for the electron- and hole-doped $\mathrm{CuO}_{2}$ systems could be the source of the strong electron-hole asymmetry in the magnetic phase diagram 16,34. 
The electron-hole asymmetry arises naturally in the three-band model [7, 12,18,28]. Not only the signs of the hopping integrals, but also the orders of the elementary processes, that lead to the effective hopping, differ for the electron and hole. Consideration of a simple limiting case as well as discussion of the electron-hole asymmetry and the dependence of the effective hopping integrals on the initial parameters are presented in Appendix A. Numerical results for the ranges of the effective parameters, based on the exact formulae of Ref. [28] and the approach developed in Ref. 18, are shown in Table I.

Further, we will consider these (Table I) $t^{\prime}, t^{\prime \prime}, t^{N}, t^{S}$ values and $t_{h}=2.5 \mathrm{~J}, t_{e}=-3.0 \mathrm{~J}[12,18$ as the realistic ones.

\section{SINGLE-CARRIER ENERGY SPECTRUM}

Before doing the energy spectrum calculation let us discuss the sense of the constrained Fermi operators $\tilde{c}^{\dagger}(\tilde{c})$ introduced in Eq. (1). Both the hole and electron singleband low-energy Hamiltonians derived from the threeband Hubbard model are naturally expressed in terms of the Hubbard operators at the site $i$

$$
X_{i}^{a b} \equiv|a i\rangle\langle b i|,
$$

here the states $a, b$ are either spin or singlet (vacancy) local states. Since the undoped state of the $\mathrm{CuO}_{2}$ plane is set up by the localized holes in the $\mathrm{Cu} d^{10}$ and $\mathrm{O} p^{6}$ orbitals, the additional low-energy hole forms a singlet with the local one, whereas the electron in this background is a vacancy. Therefore, it is convenient to rewrite $t, t^{\prime}$-parts of Eqs. (1), (3) as:

$$
\begin{aligned}
H_{\text {hole }}= & \sum_{\langle i j\rangle_{n}, \alpha} t_{i j}^{h} X_{i}^{s \alpha} X_{j}^{\alpha s}+\sum_{\langle i l j\rangle, \alpha \beta} t_{h}^{N} X_{i}^{s \alpha} X_{j}^{\alpha s} \hat{N}_{l} \\
& +\sum_{\langle i l j\rangle, \alpha \beta} t_{h}^{S} X_{i}^{s \alpha} X_{j}^{\beta s}\left(\boldsymbol{\sigma}_{\beta \alpha} \mathbf{S}_{l}\right) \\
H_{e l}= & \sum_{\langle i j\rangle_{n}, \alpha} t_{i j}^{e} X_{i}^{0 \alpha} X_{j}^{\alpha 0}+\sum_{\langle i l j\rangle, \alpha \beta} t_{e}^{N} X_{i}^{0 \alpha} X_{j}^{\alpha 0} \hat{N}_{l} \\
& +\sum_{\langle i l j\rangle, \alpha \beta} t_{e}^{S} X_{i}^{0 \alpha} X_{j}^{\beta 0}\left(\boldsymbol{\sigma}_{\beta \alpha} \mathbf{S}_{l}\right)
\end{aligned}
$$

where $|\alpha\rangle=|\uparrow\rangle,|\downarrow\rangle$ is a local spin state, $|s\rangle$ and $|0\rangle$ are singlet and vacancy states, respectively, $\langle i j\rangle_{n}$ denotes all essential neighbor sites. Note, that the signs of the nextnearest hopping parameters shown in Table I are related to these particular (Eq. (7)) order of the Hubbard operators. The signs of the nearest neighbor hopping are: $t_{\langle i j\rangle}^{h}>0, t_{\langle i j\rangle}^{e}<0$. The later is not essential, since for the antiferromagnetic background the shift of the quasimomentum space by the vector of the reciprocal lattice $Q=(\pi, \pi)$ changes the $\operatorname{sign}\left(t_{\langle i j\rangle}\right)$ but physically changes nothing 15.

We wish to stress that when the kinetic-energy part is of the single-band type (as in Eq.(7)), mapping of the
Hubbard operators onto the constrained Fermi operator basis is twofold.

If one prefers to retain the singlet ideology for the holedoped system, the first term of the Hamiltonian (7) can be rewritten identically as [14]:

$$
H_{\text {hole }}=\sum_{\langle i j\rangle_{n}, \alpha} t_{i j}^{h} n_{i,-\alpha}^{h} h_{i, \alpha}^{\dagger} h_{j, \alpha} n_{j,-\alpha}^{h}
$$

and the background is created by the $h_{i, \alpha}$-hole at every site. $n_{i,-\alpha}^{h}=h_{i,-\alpha}^{\dagger} h_{i,-\alpha}$ is the projection operator which project out the vacancy states ('double electron occupancy'). This representation for the electron over the hole background is:

$$
H_{e l}=-\sum_{\langle i j\rangle_{n}, \alpha} t_{i j}^{e}\left(1-n_{i,-\alpha}^{h}\right) h_{i, \alpha}^{\dagger} h_{j, \alpha}\left(1-n_{j,-\alpha}^{h}\right)
$$

where the operators $\left(1-n_{i, \alpha}\right)$ project out the double hole occupancy. An alternative way is to consider the physical hole as a 'hole' (vacancy) in the upper Hubbard band 35, 36. and the physical electron as the 'particle' (singlet) in the lower one. This freedom in choosing is not connected with the initial orbital structure of the $\mathrm{CuO}_{2}$ plane, but follows from the algebra of the Hubbard operators in Eq.(7). Hence, Eq.(9) for an extra electron on the electron background can be written as:

$$
H_{e l}=\sum_{\langle i j\rangle_{n}, \alpha} t_{i j}^{e} n_{i,-\alpha}^{e} e_{i, \alpha}^{\dagger} e_{j, \alpha} n_{j,-\alpha}^{e}
$$

At the first glance, it would seem that the choice of the 'particle' (Eq.(10)) or 'vacancy' (Eq.(9)) language leads to the change of the sign of the quasiparticle energy. This is not true, since the 'vacancy' energy has the sign of $t_{i j}$ reversed compared to the 'particle' energy [15,31]. Therefore, Eqs. (9) and (10) lead to the same energy.

Thus, the explicit form of the constrained operators in Eqs. (1), (3) is: $\tilde{c}_{i, \alpha}=h_{i, \alpha} n_{i, \alpha}^{h}\left(e_{i, \alpha} n_{i, \alpha}^{e}\right)$ for the physical hole (electron) system. Hereafter, we will work with the Hamiltonian Eqs. (1), (3), constraint from Eqs. (8), (10), and parameters from Table I.

Properties of the single-particle in an antiferromagnetic background were studied in detail by many authors [4, 5, 14, 19,20,37 39] using different approaches. Their results coincide at the point that the carriers are strongly dressed by the spin waves, i.e. quasiparticles are the magnetic polarons of a small radius with a strongly anisotropic dispersion law and small enough quasiparticle residue. We base our calculations on the results of Ref. [14]. The suggested trial function of an extra particle has the simple form [14]:

$$
\begin{aligned}
\psi_{\mathbf{k}, \uparrow}^{\dagger}= & \frac{1}{\sqrt{N / 2}} \sum_{n \in \downarrow}\left(\nu_{\mathbf{k}} c_{n, \uparrow}^{\dagger}\right. \\
& \left.+S_{n}^{\dagger} \sum_{\delta} \mu_{\mathbf{k}, \delta} c_{n+\delta, \downarrow}^{\dagger}\right) \exp \left(i \mathbf{k r}_{n}\right)
\end{aligned}
$$


valid also for $\psi_{\mathbf{k}, \downarrow}^{\dagger}$ after changing $\uparrow \Leftrightarrow \downarrow$ and $S^{+} \Leftrightarrow S^{-}$. $n \in \uparrow(\downarrow)$ means the site $n$ of the spin-up (-down) sublattice, $\delta$ is the unit vector to the nearest neighbor site. The explicit expressions for $\nu_{\mathbf{k}}$ and $\mu_{\mathbf{k}, \delta}$ are given in Appendix B. From the string picture point of view this simplest ansatz (11) consists of the 'bare' particle and four shortest 'strings' of the lattice constant length. It was shown 14,40 42 that the one-hole dispersion as well as two-hole contact and long-range interactions, and even many-hole properties of the $t-J$ model are quantitatively well described using the above ansatz.

Consideration of the generalized $t^{\prime}$-terms (Eq.(3)) does not require any changes in the trial function (11). Roughly, this is due to the following reasons: (i) the $t^{\prime}$ terms lead to the motion of the particle over one sublattice without distortion of the spin background, that enables 'bare' particle to propagate freely; (ii) the motion of the 'dressed' particle (with a string) mainly leads to longer strings, whose contribution to the energy is of the order of $\frac{t_{\text {eff }}^{\prime}}{t}$ and parametrically small at $t \simeq 3 \mathrm{~J}$. Hence, the expansion of the ansatz makes a small decrease in the energy, since the part of the ansatz (11), which would be mainly affected by the $t^{\prime}$-Hamiltonian (3), is the 'bare' one. The last statement will be demonstrated below.

As was noted, the $t^{\prime}$-induced transitions occur in one sublattice, therefore a good preliminary consideration of the role of each term in Hamiltonian (3) can be done for the Ising background. It allows us to find easily the leading contribution to the band minima shifts, bandwidths, and effective masses. The $\hat{N}$ and $\mathbf{S}$ operators in (3) should be replaced with $\langle\hat{N}\rangle=1,\langle\mathbf{S}\rangle=\left\langle S^{z}\right\rangle= \pm 1 / 2$. The eigenenergy of the magnetic polaron (11) is

$$
\begin{aligned}
\epsilon_{\mathbf{k}} & =\frac{\left\langle\psi_{\mathbf{k}}\left|\left(H_{t-J}+H_{t^{\prime}}\right)\right| \psi_{\mathbf{k}}\right\rangle}{\left\langle\psi_{\mathbf{k}} \mid \psi_{\mathbf{k}}\right\rangle} \simeq E_{0}+\beta_{1} \gamma_{\mathbf{k}}^{2}+\beta_{2}\left(\gamma_{\mathbf{k}}^{-}\right)^{2} \\
\beta_{1} & =4 \nu^{2}\left(t^{\prime}+2 t^{\prime \prime}+4 t^{N}-2 t^{S}\right) \\
\beta_{2} & =4 \nu^{2}\left(2 t^{\prime \prime}-t^{\prime}\right) \\
\gamma_{\mathbf{k}} & =1 / 2\left(\cos \left(k_{x}\right)+\cos \left(k_{y}\right)\right) \\
\gamma_{\mathbf{k}}^{-} & =1 / 2\left(\cos \left(k_{x}\right)-\cos \left(k_{y}\right)\right),
\end{aligned}
$$

where we omitted all high-order $\left(t_{\text {eff }}^{\prime} / t\right)$ terms. $\mathrm{E}_{0}$ is the depth of the band, $\beta_{1}, \beta_{2}$ are the inverse masses in the directions ortogonal and parallel to the magnetic Brillouin zone boundary, respectively. Since for the Ising case a particle of the pure $t$ - $J$ model is dispersionless [43], $\nu$ and $\mu$ are constants and at the realistic $t \simeq(2-3) J$ $|\nu|^{2} \simeq \frac{1}{2},|\mu|^{2} \simeq \frac{1}{8}$. Expression (12) for the energy coincides almost exactly with that obtained for the free spinless fermions [26]. The main difference is the presence of the weight of the 'bare' particle $|\nu|^{2}$ in Eq. (12). In the realistic region of $t, t^{\prime}, t^{\prime \prime}$ etc $E_{0}$ is very close to its $t-J_{z}$ model value $E_{0} \simeq-2 t$.

Two notes should be done. Firstly, the $\beta_{1}$-term (12) represents the dispersion which is degenerate along the $(\pi, 0)-(0, \pi)$ line, whereas the $\beta_{2}$-term lifts out this degeneracy, placing the minima at the $( \pm \pi / 2, \pm \pi / 2)$ (if $\left.\beta_{2}>0\right)$ or $( \pm \pi, 0),(0, \pm \pi)$ (if $\left.\beta_{2}<0\right)$ points. The difference $\Delta_{(0, \pi)-(\pi / 2, \pi / 2)} \equiv \beta_{2}$ is proportional only to the 'first-order' terms $t^{\prime}, t^{\prime \prime} 12$ and does not depend on the second-order $t^{N}, t^{S}$ terms. Secondly, one can see from Eq. (12) that in the case $t^{N}>0, t^{S}<0$ and $\operatorname{sign}\left(t^{\prime}\right)=-\operatorname{sign}\left(t^{\prime \prime}\right) \beta_{1}$ and bandwidth $W\left(=\beta_{1}\right.$ or $\left.\beta_{1}-\beta_{2}\right)$ are mostly determined by a few $\left(2 t^{N}-t^{S}\right)$.

The above qualitative calculation for the simple Ising background results in conclusions, which remain valid for the Néel case. The energy difference between the $(\pi / 2, \pi / 2)$ and $(\pi, 0)$ points, which is the crucial value for the various calculations, has different signs for the hole- and electron-doped systems and is not small for both cases. It means that if the single-particle picture is valid for the finite doping regime, the quasiparticle Fermisurface will be located (up to the high enough doping level) near the points $( \pm \pi / 2, \pm \pi / 2)$ and $( \pm \pi, 0),(0, \pm \pi)$ for the hole- and electron-doped systems, respectively.

Also, it can be shown from Eq. (12) that, at least for the upper limit of the $t^{\prime}, t^{\prime \prime}, t^{S}, t^{N}$ hopping parameters (Table I), the bandwidths are large enough. Thus, one would expect decrease of the DOS compared to the pure $t$ - $J$ model one.

Finally, the role of the 'bare' particle for the considered simple case is crucial, since the 'dressed' part cannot propagate freely in the absence of the spin fluctuations. Therefore, the weight of the bare part in the trial wave function is the subject of the prime interest. As was noted $n^{\text {bare }}=\nu^{2} \simeq 0.5$, that is larger than in other works 37 39] where $\tilde{n}^{\text {bare }}=\tilde{\nu}^{2} \simeq 0.35-0.41$. For $n^{1 \text { string }}=4|\mu|^{2} \simeq 0.42$ the agreement is better: $\tilde{n}^{1 s t r i n g} \simeq 0.44$. This discrepancy is due to the transfer of the weight from the rest of the exact particle wave function (with the infinite number of strings) to the weight of the 'bare' particle in the approximate ansatz (11). Thus, our main approximation lies not in the 'shortness' of the ansatz, but in the overestimation of the bare particle weight. This problem can be overcome by the simple renormalization of $\nu^{2}$ to $\tilde{\nu}^{2}$.

Let us discuss now what changes of the above results can be expected for the Néel background. The main changes arise from the fact that due to the spin fluctuations the pure $t-J$ model particle is given the possibility to propagate. Evidently, this will provide additions to $\beta_{1}, \beta_{2}$ and the bandwidth $W$. Also, the 'dressed' part will lead to some coherent transitions. Due to the more complex structure of the matrix elements of the Hamiltonian (11), (3) for the Néel background, the simple tight-binding relations between $\beta_{1}, \beta_{2}$ and $W$ no longer hold.

The pure $t-J$ dispersion law [14, 41] is:

$$
\begin{aligned}
\epsilon^{t-J}(\mathbf{k})= & 2 J-\sqrt{(0.66 J)^{2}+4.56 t^{2}-2.8 t^{2} \gamma_{\mathbf{k}}^{2}} \\
& +0.01|t|\left(\gamma_{\mathbf{k}}^{-}\right)^{2} \\
& \simeq E_{0}^{t-J}+\beta_{1}^{t-J} \gamma_{\mathbf{k}}^{2}+\beta_{2}^{t-J}\left(\gamma_{\mathbf{k}}^{-}\right)^{2}
\end{aligned}
$$

where $\beta_{1}^{t-J} \simeq+0.65|t|$ and $\beta_{2}^{t-J} \simeq+0.01|t|$. Due to the $\mathbf{k}$-dependence of the trial wave function components $\nu_{\mathbf{k}}, \mu_{\mathbf{k}}$ and the nonzero matrix elements for the string 
components, the first order contribution to the energy from the $t^{\prime}$-Hamiltonian (3) contains extra terms with the highest powers of $\gamma_{\mathbf{k}}^{2}$ :

$$
\begin{aligned}
& \epsilon(\mathbf{k})=\epsilon^{t-J}(\mathbf{k})+\delta \epsilon^{t^{\prime}}(\mathbf{k}) \\
& \delta \epsilon^{t^{\prime}}(\mathbf{k})= \delta E_{0}+\delta \beta_{1, \mathbf{k}} \gamma_{\mathbf{k}}^{2}+\delta \beta_{2, \mathbf{k}}\left(\gamma_{\mathbf{k}}^{-}\right)^{2} \\
&+A_{\mathbf{k}} \gamma_{\mathbf{k}}^{4}+B_{\mathbf{k}} \gamma_{\mathbf{k}}^{2}\left(\gamma_{\mathbf{k}}^{-}\right)^{2}
\end{aligned}
$$

where the $\delta \beta_{1, \mathbf{k}}, \delta \beta_{2, \mathbf{k}}, A_{\mathbf{k}}$ and $B_{\mathbf{k}}$ coefficients weakly depend on $\mathbf{k}$ through the $\left|\nu_{\mathbf{k}}\right|^{2}$ and $\left|\mu_{\mathbf{k}}\right|^{2}$ quantities (see Appendix B). As was discussed earlier, the highest-order corrections to the energy from Eq. (3) are of the order of $\frac{t_{\text {eff }}^{\prime}}{t}$, and for the realistic $t / J \simeq(2-3)$ and $t^{\prime}$ from Table I can be neglected $\left(\sim \frac{1}{10}\right)$. Since $\left|\nu_{\mathbf{k}}\right|^{2}$ is almost constant, the renormalization to the 'normal' $|\tilde{\nu}|^{2}$ can be accomplished by the simple replacing $\left|\nu_{\pi / 2, \pi / 2}\right|^{2} \simeq\left|\nu_{\pi, 0}\right|^{2} \simeq$ $\left|\nu_{0,0}\right|^{2} \Rightarrow|\tilde{\nu}|^{2} \simeq 0.35$.

Figures 1-1 present our results for the hole and electron dispersions. Contour plot in Fig. 1 shows the characteristic feature of the hole dispersion in the whole Brillouin zone. The magnetic Brillouin zone boundary as well as the $\Gamma(0,0) \rightarrow M(\pi / 2, \pi / 2) \rightarrow Z(\pi, \pi) \rightarrow Y(0, \pi) \rightarrow \Gamma$ and $Y \rightarrow M \rightarrow X(\pi, 0)$ directions are shown. Figs. 24 show the dispersions along the $\Gamma \rightarrow M \rightarrow Z \rightarrow Y \rightarrow \Gamma$, and $Y \rightarrow M \rightarrow X$ lines. Fig. 2 demonstrates the characteristic $t-t^{\prime}-J$ hole dispersion (solid line) for the average parameters from Table I, pure $t-J$ model hole dispersion (dashed line), and pure $t^{\prime}-J$-dispersion $t \equiv 0$ (dotted line). This qualitative picture clearly demonstrates that due to the $t^{\prime}$-terms the system becomes 'less strongly correlated' because of the 'liberating' of the bare carrier. Figs. 3 and 4 show dispersions along the same lines. Fig. 3 describes the hole-doped system, and Fig. At the electron-doped one. Solid curves are related to the upper and lower limit for the $t^{\prime}, t^{\prime \prime}, t^{N}, t^{S}$ parameters from Table I, the dotted satellites are related to the same parameters for the renormalized bare carrier weight (up to $0.35)$. The dashed lines demonstrate the pure $t-J$ model dispersions.

Our calculations of the $t^{\prime}$-part (14) in the dispersion demonstrate that the 'bare' particle contribution for the Néel case is very close to the results for the Ising background and that the 'dressed' particle contribution plays a minor role $(<0.2)$ compared to the 'bare' one. Using the data from Table I, the variations of the inverse mass values and the bandwidths for the hole and electron are found as follows:

$$
\begin{aligned}
& \beta_{1}^{h}=(3.8-5.3) J, \quad \beta_{1}^{e}=(3.5-5.0) J \\
& \beta_{2}^{h}=(1.3-3.1) J, \quad \beta_{2}^{e}=-(0.7-1.6) J \\
& W^{h}=(5.1-7.1) J, \quad W^{e}=(5.2-8.4) J
\end{aligned}
$$

In spite of some changes in the inverse mass values (15), the features discussed earlier for the Ising case are still valid. Thus, the shifts of the band minima are rather large, the bandwidths are substantially wider as com- pared to those in the $t-J$ model, and the role of the bare particle weight remains the most important.

One of the unsolved problem of the $t-J$ type models is: whether the free-particle approximation work for the doped systems 31,38,44. Therefore, the extension of the single-particle calculation onto the case of finite doping indeed requires justifications. Some of them can be found in Refs. 20,31,38. We simply accept the free particle picture and have calculated the chemical potential as the function of concentration for the dispersion law (13), (14). From Figs. 11-14 it is clear that the ground state is $( \pm \pi / 2, \pm \pi / 2)$ for the hole, and $(0, \pm \pi)$, $( \pm \pi, 0)$ for the electron. Both minima are not shallow: $\left|\Delta_{(0, \pi)-(\pi / 2, \pi / 2)}\right| \sim J$. At the same time, the bandwidths are larger than $t-J$ ones $\left(W^{t-J} \sim 2 J\right)$, and the accumulation of the DOS at low energy is smaller than for the $t$ - $J$ model case. This point may be of interest in view of intensive discussion of the possible Van Hove singularity at the optimal doping [45]. The above mentioned two different tendencies to increase the minima shifts and to decrease the DOS require a quantitative consideration. Figs. 5 and 6 show our results for the DOS versus energy $E / J$ and versus concentration $\delta$, respectively. One can see that in spite of the DOS lowering it remains rather large at low energies (since $(3-8) \mathrm{J}$ $\ll 8 t$ ), and the peaks in the DOS shift to higher concentrations $\left(\delta_{\max } \sim 0.4-0.5\right)$, compared to the $t-J$ case $\left(\delta_{\max } \sim 0.1-0.2\right)$.

\section{CONCLUSIONS}

To summarize, in this paper we have studied the microscopically derived extended type of the $t-t^{\prime}-J$ model. Previous investigations of the three-band model allowed us to establish the necessary next-neighbor terms for the low-energy single-band model. In this work the efficient scheme, developed previously for the calculation of the $t$ $J$ model parameters of the real $\mathrm{CuO}_{2}$ systems, has been applied to the effective $t^{\prime}$-terms calculation. This has enabled us to determine numerical ranges for all $t^{\prime}$-terms both for the electron- and hole-doped systems.

We also have performed simple calculations of the single-particle dispersion using the variational approach to the spin-polaron problem. Both types of doping have been considered within the above mentioned realistic values of the $t, t_{\text {eff }}^{\prime}$ parameters. The importance of all $t_{\text {eff }}^{\prime}$ terms has been clearly demonstrated: the $t^{N}, t^{S}$ terms of the second-order origin are responsible for the widening of the bandwidth, and the $t^{\prime}, t^{\prime \prime}$-terms arising from the O-O hopping bring about the shift of the groundstate minima. It has been found that the minima shifts are large enough $(\sim J)$ and have opposite signs for the hole and electron systems. Also, the bandwidths have been found to be $1.5-4.0$ times larger than those in the pure $t-J$ model.

An investigation of the finite doping regime for the 
simple free-particle approximation has been carried out. The Van Hove peak in the DOS has been found to decrease and move to a higher doping level from its $t$ - $J$ model position.

\section{Acknowledgments}

It is our pleasure to thank A. Barabanov, L. Maksimov and O. Sushkov for initiative discussions and M. Avignon for helpful conversation. We are grateful to O. A. Starykh for instructive discussion and sending their results to us prior to publication. This work was supported in part by the Russian Foundation for Fundamental Researches, Grant No 94-02-03235; International Science Foundation established by G. Soros, Grant No J2M100; The Competition Center for Natural Sciences at St.-Peterburg State University, Grant No 94-5.1-1060; Council on Superconductivity of Russian Academy of Sciences, Grant No 93197; by the Scientific-Technical Program "High - Temperature Superconductivity" as part of the state program "Universities as Centers for Fundamental Research". The work of one of us (A.L.C.) has been supported by a fellowship of INTAS Grant 93-2492 and is carried out within the research program of International Center for Fundamental Physics in Moscow.

\section{APPENDIX A:}

It will be useful for the future discussion to consider the simple limiting case of the three-band model, when the $\mathrm{Cu}-\mathrm{O}$ hopping is much less than both the $\mathrm{Cu}-\mathrm{Cu}$ Coulomb repulsion $\mathrm{U}_{d}$ and $\mathrm{Cu}-\mathrm{O}$ levels splitting $\Delta\left(t_{p d} \ll\right.$ $\left.\mathrm{U}_{d}, \Delta\right)$.

The additional hole moves over the spin background as a Zhang-Rice (ZR) singlet [6], whereas the electron moves as a vacancy. Corresponding hopping integrals in the lowest order of $t_{p d} / \Delta$ are:

$$
\begin{aligned}
& t_{h}=0.53 \frac{t_{p d}^{2}}{\tilde{\Delta}}+0.275 t_{p p}, \quad t_{e}=-\left(1.06+2.03 \frac{t_{p p}}{\tilde{\Delta}}\right) \frac{t_{p d}^{2}}{\tilde{\Delta}}, \\
& t_{h}^{\prime}=0.09 \frac{t_{p d}^{2}}{\tilde{\Delta}}-0.124 t_{p p}, \quad t_{e}^{\prime}=-\left(0.18-0.92 \frac{t_{p p}}{\tilde{\Delta}}\right) \frac{t_{p d}^{2}}{\tilde{\Delta}}, \\
& t_{h}^{\prime \prime}=0.053 \frac{t_{p d}^{2}}{\tilde{\Delta}}+0.062 t_{p p}, \quad t_{e}^{\prime \prime}=-\left(0.105+0.46 \frac{t_{p p}}{\tilde{\Delta}}\right) \frac{t_{p d}^{2}}{\tilde{\Delta}} \\
& \tilde{\Delta}=\Delta-1.45 t_{p p} .
\end{aligned}
$$

The first two expressions for the hole hopping integrals coincide with those in the work by Jefferson et al [8], except the signs. The signs are opposite for the following reasons.

In calculation of the transfer amplitudes of the $t$ - $J$ model singlet or vacancy from the three-band model, it is convenient to construct the basis of the wave functions as the direct product of the states at the sites:
$\left(\hat{A}_{1}^{\dagger}|0\rangle_{1} \otimes \hat{A}_{2}^{\dagger}|0\rangle_{2} \otimes \ldots\right)$. The $\hat{A}_{i}^{\dagger}$ operators can be considered as the Hubbard operators at the site $i$, which create the singlet or spin state over the vacuum state $|0\rangle_{i}$. Namely, the two-site wave function of the form $|s\rangle_{i} \otimes|\uparrow\rangle_{j}$ has been used for the calculation of the singlet $\left(|s\rangle_{i}\right)$ hopping integrals (see Refs. [7, 8, 28]). This convenience is due to the complex structure of the three-band low-energy states. For example, ZR singlet consists of the linear combination of a copper singlet (double copper occupancy), an oxygen one (double oxygen occupancy), and a copper-oxygen one. This state is hardly expressed in the terms of the Fermi creation operator which acts on the spin background. However, the use of the 'directproduct' basis is incorrect when more than one Hubbard $\hat{A}_{i}^{\dagger}$ operators are of the Fermi type. This is evident from their anticommutativity. In the other words, the wave function of the half-filled background (one fermion per site) cannot be uniquely determined in this basis. Formally, the two-site wave function $|s\rangle_{i} \otimes|\uparrow\rangle_{j}$ is determined correctly since the singlet $|s\rangle_{i}$ is the boson. Nevertheless, for the matrix elements of the hopping Hamiltonian, which consists of the creation and annihilation operators of an additional fermion, one gets:

$$
\begin{aligned}
& t_{i j}\left\langle\left.\uparrow\right|_{i} \otimes\left\langle\left. s\right|_{j}\left[(|s\rangle\langle\uparrow|)_{j} \cdot(|\uparrow\rangle\langle s|)_{i}\right] \mid s\right\rangle_{i} \otimes \mid \uparrow\right\rangle_{j} \\
\equiv & t_{i j}\left\langle\left.\uparrow\right|_{i} \otimes\left\langle\left.\uparrow\right|_{j} \cdot \mid \uparrow\right\rangle_{i} \otimes \mid \uparrow\right\rangle_{j} .
\end{aligned}
$$

Thus, the matrix element reduces to the projection of the one half-filled state to the other and hence has an uncertain sign. Therefore, to avoid this uncertainty one has to use a more conventional basis, namely: $c_{i}^{\dagger}|g s\rangle$, where $|g s\rangle$ means the groundstate (one fermion per site), and $c_{i}^{\dagger}$ is the creation operator of the additional fermion. This careful approach leads to the signs of the hopping amplitudes as presented in Eq. A1 and in Table I. Note that this difference of the bases is absent for the single fermion in the lattice or for particles obeying Bose statistics.

To obtain Eq. (A1) we have used the general expressions for $t_{i j}$ from our previous works 12,28 .

Approximate expressions (A1) for $t, t^{\prime}$ and $t^{\prime \prime}$ are good enough for large and even moderate $\Delta$. Thus, our calculations of the $t, t^{\prime}, t^{\prime \prime}$ realistic values for the $\mathrm{CuO}_{2}$ plane, show that $\left|t^{\prime}\right|<\left|t^{\prime \prime}\right|$ at least for small values of $V_{p d}(\mathrm{Cu}-\mathrm{O}$ Coulomb repulsion). This is due to the partial compensation of the $\mathrm{Cu}-\mathrm{O}$ and $\mathrm{O}-\mathrm{O}$ contribution to the $t^{\prime}$-term, that can be seen in Eq.A1). It should be noted that the $t_{p p}$ contribution to the hopping of the vacancy arises only in the third order of $t_{p d} / \Delta\left(t_{p p} / \Delta\right)$. The above mentioned compensation of the $\mathrm{O}-\mathrm{O}$ and $\mathrm{Cu}-\mathrm{O}$ amplitudes for the electron $t_{e}^{\prime}$-term is even more pronounced than for the hole. The exact formulae [12] provide very small $t_{e}^{\prime}$ for $V_{p d}=0$.

To be more specific, in our calculations we have followed the idea of the narrowing of the uncertainty region for the low-energy model parameters using some experimentally observed values. This approach was developed in Ref. 18 and enabled us to calculate $t_{h}$ and $t_{e}$ for the 
real $\mathrm{CuO}_{2}$ planes. Our results for the $t^{\prime}, t^{\prime \prime}, t^{N}, t^{S}$ hopping integrals obtained in the same way for the two types of doping are shown in Table I.

We wish to stress the strong $V_{p d}$-dependence of $t^{\prime}$ both for the hole and electron carriers. This $t^{\prime}\left(V_{p d}\right)$ dependence can be easily understood. The rising of $V_{p d}$ requires the lowering of the $\Delta$ for the fixed values of the observable quantities. Thus, the occupation number of oxygen sites also rises, which immediately leads to the growing of the $t_{p p}$ contribution. Note, that due to the smaller role of the oxygen degrees of freedom for the electron, its hopping integrals are less varying than those for hole. This is in agreement with the cluster calculations by Eskes and Sawatzky [7,32]. In our three-band model calculations we used the experimental values of $J=0.14$ and $J=0.17 \mathrm{eV}$ (lanthanum and yttrium systems).

\section{APPENDIX B:}

Explicit expressions for the components $\nu_{\mathbf{k}}, \mu_{\mathbf{k}}$ [14] are:

$$
\begin{aligned}
\nu_{\mathbf{k}} & =\frac{1}{2}\left[\frac{\Delta_{0}+2 S_{\mathbf{k}}}{X S_{\mathbf{k}}}\right]^{1 / 2}, \\
\mu_{\mathbf{k}, \delta} & =\mu_{\mathbf{k}}^{1}+\mu_{\mathbf{k}}^{2} \gamma_{\mathbf{k}} e^{i \mathbf{k} \delta} \\
& =\frac{t}{\left[Y S_{\mathbf{k}}\left(\Delta_{0}+2 S_{\mathbf{k}}\right)\right]^{1 / 2}}\left[(1+v)-(u+v) \gamma_{\mathbf{k}} e^{i \mathbf{k} \delta}\right]
\end{aligned}
$$

where parameters $X, Y, u$, and $v$ are expressed in terms of ground state correlators [14] and for the Néel state are: $\Delta_{0}=1.33 \mathrm{~J}, X=0.8, Y=0.72, u=0.42, v=0.12$. $S_{\mathbf{k}} \equiv \epsilon^{t-J}(\mathbf{k})$ is the pure $t-J$ model dispersion (13). Since $S_{\mathbf{k}} \sim|t|$ at $t>J, \nu_{\mathbf{k}}$ and $\mu_{\mathbf{k}}^{1}, \mu_{\mathbf{k}}^{2}$ are weakly varying functions of $\mathbf{k}$.

Explicit expressions for the $\delta \beta_{1, \mathbf{k}}, \delta \beta_{2, \mathbf{k}}, A_{\mathbf{k}}, B_{\mathbf{k}}$ coefficients (Eq.(14)) are:

$$
\begin{aligned}
\delta \beta_{1, \mathbf{k}} & =\nu_{\mathbf{k}}^{2} C_{1}^{1}+\left(\mu_{\mathbf{k}}^{1}\right)^{2} C_{1}^{2}+\left(\mu_{\mathbf{k}}^{2}\right)^{2} C_{1}^{3}+\mu_{\mathbf{k}}^{1} \mu_{\mathbf{k}}^{2} C_{1}^{4}, \\
\delta \beta_{2, \mathbf{k}} & =\nu_{\mathbf{k}}^{2} C_{2}^{1}+\left(\mu_{\mathbf{k}}^{1}\right)^{2} C_{2}^{2}+\left(\mu_{\mathbf{k}}^{2}\right)^{2} C_{2}^{3}+\mu_{\mathbf{k}}^{1} \mu_{\mathbf{k}}^{2} C_{2}^{4}, \\
A_{\mathbf{k}} & =\nu_{\mathbf{k}}^{2} C_{3}^{1}+\left(\mu_{\mathbf{k}}^{1}\right)^{2} C_{3}^{2}+\left(\mu_{\mathbf{k}}^{2}\right)^{2} C_{3}^{3}+\mu_{\mathbf{k}}^{1} \mu_{\mathbf{k}}^{2} C_{3}^{4}, \\
B_{\mathbf{k}} & =\nu_{\mathbf{k}}^{2} C_{4}^{1}+\left(\mu_{\mathbf{k}}^{1}\right)^{2} C_{4}^{2}+\left(\mu_{\mathbf{k}}^{2}\right)^{2} C_{4}^{3}+\mu_{\mathbf{k}}^{1} \mu_{\mathbf{k}}^{2} C_{4}^{4},
\end{aligned}
$$

where

$$
\begin{aligned}
& C_{1}^{1}=3 t^{\prime}+6 t^{\prime \prime}+12 t^{N}-8.4 t^{S}, \\
& C_{1}^{2}=-5.8 t^{\prime \prime}-5.8 t^{N}-1.6 t^{S}, \\
& C_{1}^{3}=-8.2 t^{\prime \prime}-8.2 t^{N}+2.9 t^{S}, \\
& C_{1}^{4}=9.8 t^{\prime}-6.7 t^{\prime \prime}+12.8 t^{N}+3 t^{S}, \\
& C_{2}^{1}=-3 t^{\prime}+6 t^{\prime \prime}, \\
& C_{2}^{2}=-1.5 t^{S}, C_{2}^{3}=C_{2}^{4}=C_{3}^{1}=C_{4}^{1}=0, \\
& C_{3}^{2}=5.8 t^{\prime}+11.5 t^{\prime \prime}+23 t^{N}-12.2 t^{S}, \\
& C_{3}^{3}=10.6 t^{\prime}+16.4 t^{\prime \prime}+37.7 t^{N}-15.1 t^{S}, \\
& C_{3}^{4}=11.5 t^{\prime}+23 t^{\prime \prime}+23 t^{N}-30.4 t^{S}, \\
& C_{4}^{2}=-5.8 t^{\prime}+11.5 t^{\prime \prime}+4.6 t^{S},
\end{aligned}
$$

$$
\begin{aligned}
& C_{4}^{3}=-10.6 t^{\prime}+16.4 t^{\prime \prime}-4.9 t^{N}+3.5 t^{S}, \\
& C_{4}^{4}=-11.5 t^{\prime}+23 t^{\prime \prime},
\end{aligned}
$$

Numbers in Eq. (B3) result from $\left\langle S_{i}^{z} S_{j}^{z}\right\rangle,\left\langle S_{i}^{+} S_{j}^{-}\right\rangle$and other spin correlators for various neighbor sites.

[1] V. J. Emery, Phys. Rev. Lett. 58, 2794 (1987).

[2] C. M. Varma, S. Schmitt-Rink, and E. Abrahams, Solid State Commun. 62, 681 (1987).

[3] Yu. B. Gaididei and V. M. Loktev, Phys. Stat. Sol. (b) 147, 307 (1988).

[4] A. F. Barabanov, R. O. Kuzian and L. A. Maksimov, J. Phys.: Condensed Matter 3, 9129 (1991).

[5] A. F. Barabanov, L. A. Maksimov, and G. V. Uimin, Sov. Phys. JETP 69, 371 (1989).

[6] F. C. Zhang and T. M. Rice, Phys. Rev. B 37, 3759 (1988).

[7] H. Eskes, G. A. Sawatzky, and L. F. Feiner, Physica C 160, 424 (1989); H. Eskes, L. H. Tjeng, and G. A. Sawatzky, Phys. Rev. B 41, 288 (1990); H. Eskes and G. A. Sawatzky, ibid. 43, 119 (1991).

[8] J. H. Jefferson, H. Eskes, and L. F. Feiner, Phys. Rev. B 45, 7959 (1992).

[9] M. E. Simon and A. A. Aligia, Phys. Rev. B 48, 7471 (1993);

[10] S. V. Lovtsov and V. Yu. Yushankhai, Physica C 179, 159 (1991).

[11] V. I. Belinicher and A. L. Chernyshev, Phys. Rev. B 47, 390 (1993).

[12] V. I. Belinicher and A. L. Chernyshev, Physica C 213, 298 (1993).

[13] E. Dagotto, A. Nazarenko, and M. Boninsegni, Phys. Rev. Lett. 73, 728 (1994).

[14] O. P. Sushkov, Sol. State. Commun. 83, 303 (1992).

[15] A. V. Chubukov and K. A. Musaelian, Phys. Rev. B 50, 6238 (1994).

[16] R. J. Gooding, K. J. E. Vos, and P. V. Leung, Phys. Rev. B 49, 4119 (1994).

[17] T. Tohyama and S. Maekava, Phys. Rev. B 49, 3596 (1994).

[18] V. I. Belinicher, A. L. Chernyshev, and L. V. Popovich, Phys. Rev. B 50, 13768 (1994).

[19] A. Auerbach and B. E. Larson, Phys. Rev. Lett. 66, 2262 (1991).

[20] E. Dagotto, Int. Jour. of Mod. Phys. B 5, 907 (1991) and references therein.

[21] P. A. Lee, Phys. Rev. Lett. 63, 680 (1989).

[22] A. Nazarenko, K. J. E. Vos, S. Haas, E. Dagotto, and R. J. Gooding,, Phys. Rev. B 51, 8676 (1995).

[23] Mark S. Hybertsen, E. B. Stechel, M. Schluter, and D. R. Jennison, Phys. Rev. B 41, 11068 (1990).

[24] G. C. Psaltakis, Phys. Rev. B 45, 539 (1992); G. C. Psaltakis and N. Papanicolaou, ibid. 48, 456 (1993).

[25] J. Bala, A. M. Oles, and J. Zaanen (unpublished). 
[26] F. P. Onufrieva, V. P. Kushnir, and B. P. Toperverg, Phys. Rev. B 50, 12935 (1994).

[27] O. A. Starykh, O. F. A. Bonfim, and G. R. Reiter, (to be published in Phys. Rev. B, Brief Reports, code number BFS571).

[28] V. I. Belinicher and A. L. Chernyshev, Phys. Rev. B 49, 9746 (1994).

[29] S. Trugman, Phys. Rev. B 41, 892 (1990).

[30] R. Eder and K. W. Becker, Z. Phys. B - Condensed Matter 78, 219 (1990).

[31] R. J. Gooding, K. J. E. Vos, and P. V. Leung, Phys. Rev. B 50, 12866 (1994).

[32] H. Eskes and G. A. Sawatzky, Phys. Rev. B 44, 9656 (1991).

[33] B. S. Shastry, Phys. Rev. Lett. 63, 1288 (1989).

[34] A. V. Chubukov and K. A. Musaelian (unpublished).

[35] C. L. Kane, P.A. Lee, and N. Read, Phys. Rev. B 39, 6880 (1989).

[36] N. M. Plakida, V. Yu. Yushankhai, and I. V. Stasyuk, Physica C 160, 80 (1989).

[37] G. Martinez and P. Horsch, Phys. Rev. B, 44, 317 (1991).

[38] R. Eder and Y. Ohta, Phys. Rev. B, 50, 10043 (1994).

[39] Z. Liu and E. Manousakis, Phys. Rev. B, 45, 2425 (1992).

[40] A. L. Chernyshev, A. V. Dotsenko, and O. P. Sushkov, Phys. Rev. B 49, 6197 (1994).

[41] M. Yu. Kuchiev and O. P. Sushkov, Physica C 218, 197 (1993).

[42] V. V. Flambaum, M. Yu. Kuchiev, and O. P. Sushkov, Physica C 227, 267 (1993).

[43] L. N. Bulaevskii, E. L. Nagaev, and D. I. Khomskii, Sov. Phys. JETP 27, 638 (1967).

[44] W. Stephan and P. Horsch, Phys. Rev. Lett. 66, 2258 (1991).

[45] E. Dagotto, A. Nazarenko, and A. Moreo, Phys. Rev. Lett. 74, 310 (1995).

\section{Figure captions}

FIG. 1. Contour plot of $\epsilon(\mathbf{k})$ for a hole. $t_{h} / J=2.5$, $t^{\prime}, t^{\prime \prime}, t^{N}, t^{S}$ are taken as the average values of the upper and lower limit from Table I. Inner square is the magnetic Brillouin zone boundary. $\Gamma(0,0), M(\pi / 2, \pi / 2), Z(\pi, \pi), Y(0, \pi)$, and $X(\pi, 0)$ points are indicated.

FIG.

2.

Dispersion curves along the lines $\Gamma \rightarrow M \rightarrow Z \rightarrow Y \rightarrow \Gamma$, and $Y \rightarrow M \rightarrow X$. Solid curve is the $t-t^{\prime}-J$ hole dispersion for the same parameters as in Fig. 1, dotted curve is the pure $t^{\prime}-J$ dispersion $(t \equiv 0)$, dashed curve is the pure $t$ - $J$ model dispersion.

FIG. 3. Dispersion curves along the lines as in Fig. 2 for a hole. Solid curves correspond to the upper and lower limits of the $t^{\prime}$-parameters (Table I). Dotted curves correspond to the same with renormalized bare carrier weight. Dashed curve is the pure $t-J$ model dispersion.
FIG. 4. Dispersion curves along the same lines as in Fig. 2 for an electron. All notations as in Fig.3.

FIG. 5. DOS vs energy $E / J$ for the pure $t-J$ model and electron and hole $t-t^{\prime}-J$ model (upper and lower limits in Table I).

FIG. 6. DOS vs concentration $\delta$.

\section{Tables}

Table I. $t^{\prime}, t^{\prime \prime}, t^{S}, t^{N}$ hopping parameters for the hole and electron. First and second rows show the lower and upper limits, respectively.

\begin{tabular}{c|cccc}
\hline & $t^{\prime} /|t|$ & $t^{\prime \prime} /|t|$ & $t^{N} /|t|$ & $t^{S} /|t|$ \\
& & & & \\
\hline hole & 0.01 & 0.12 & 0.01 & -0.07 \\
$t_{h} / J=2.5$ & -0.25 & 0.16 & 0.07 & -0.16 \\
\hline & & & & \\
\hline electron & -0.09 & -0.11 & 0.07 & -0.10 \\
$t_{e} / J=-3.0$ & 0.03 & -0.12 & 0.11 & -0.12 \\
& & & & \\
\hline
\end{tabular}




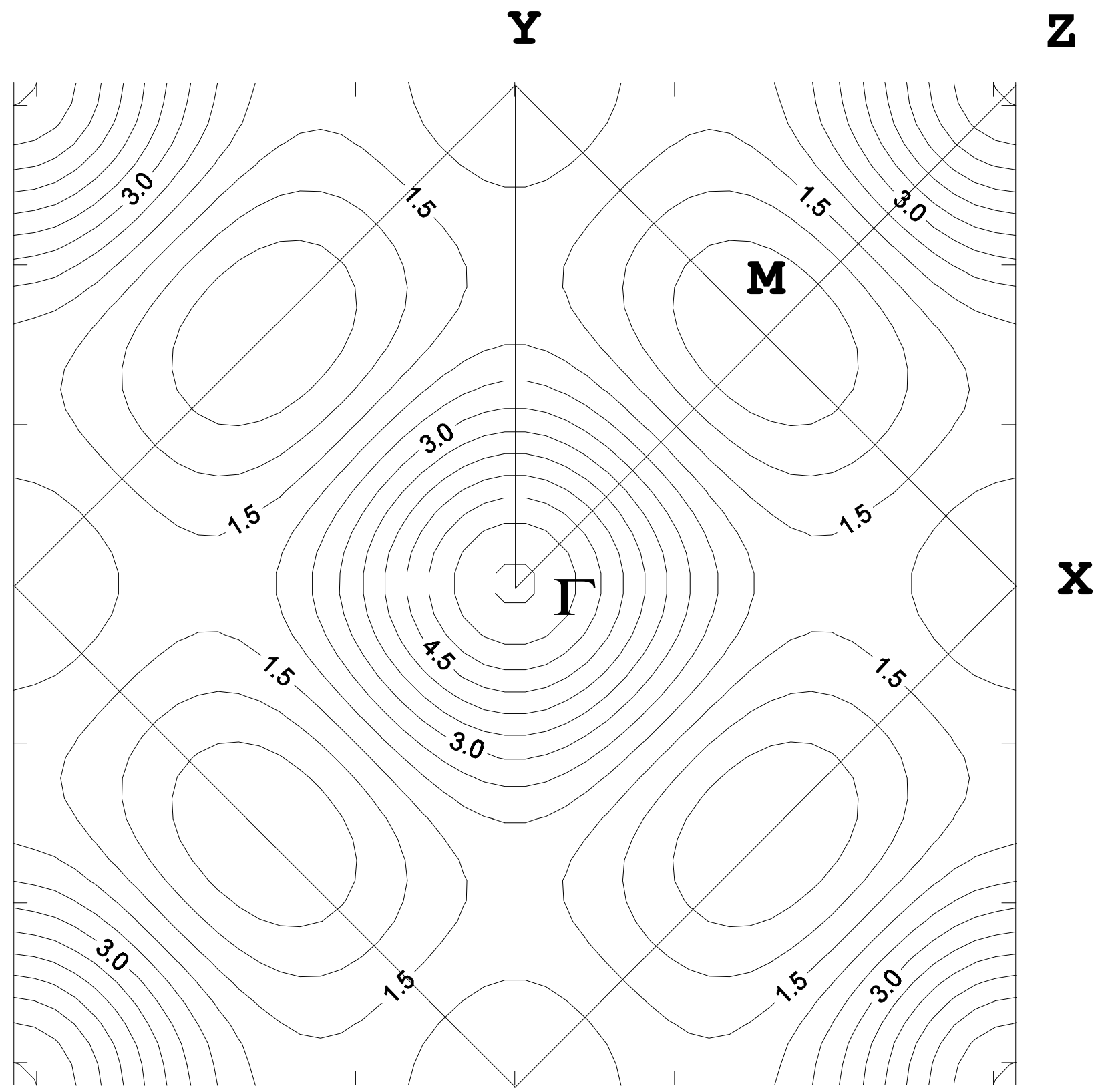

Fig. 1 
Fig. 2

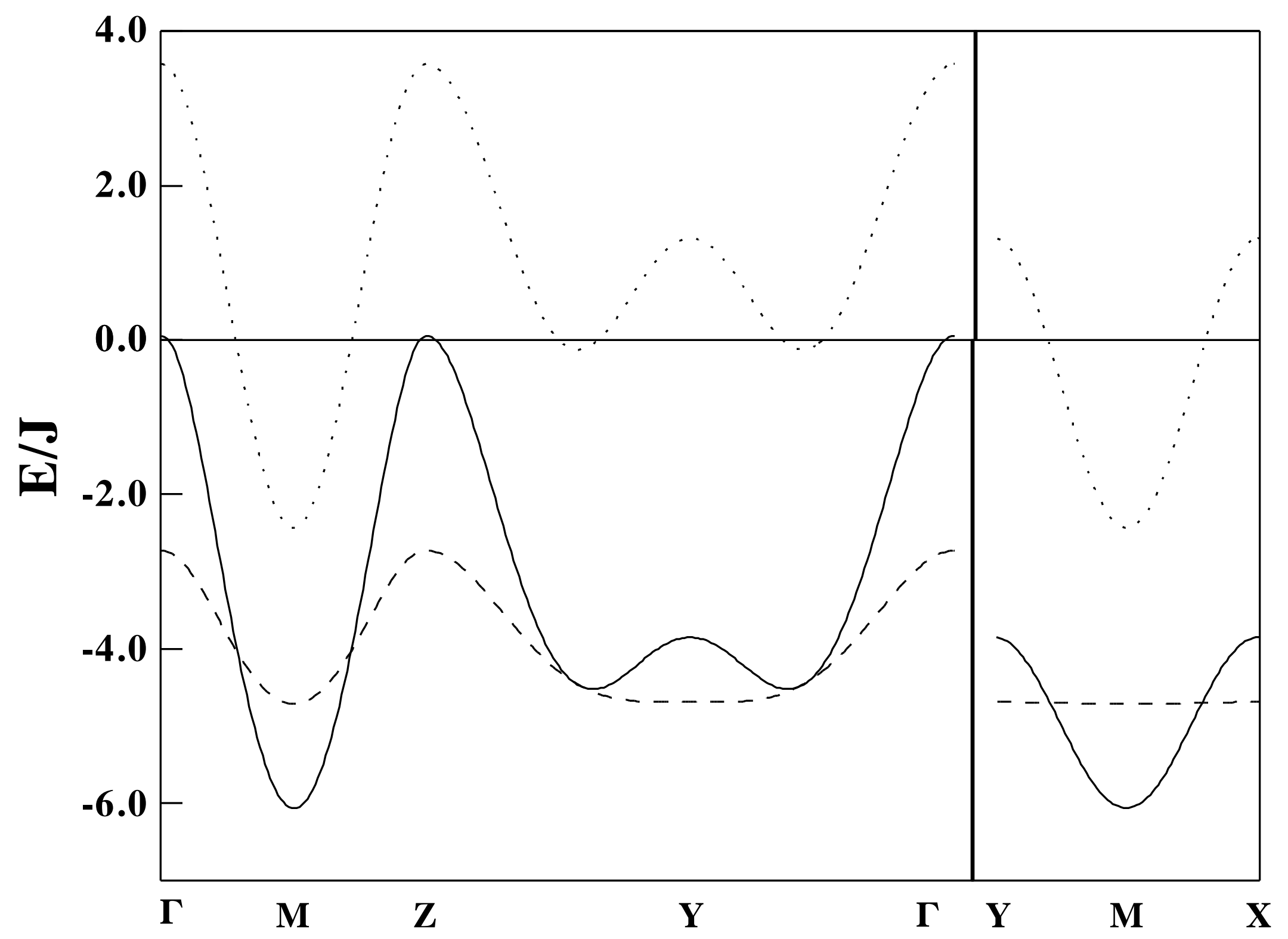


Fig. 3

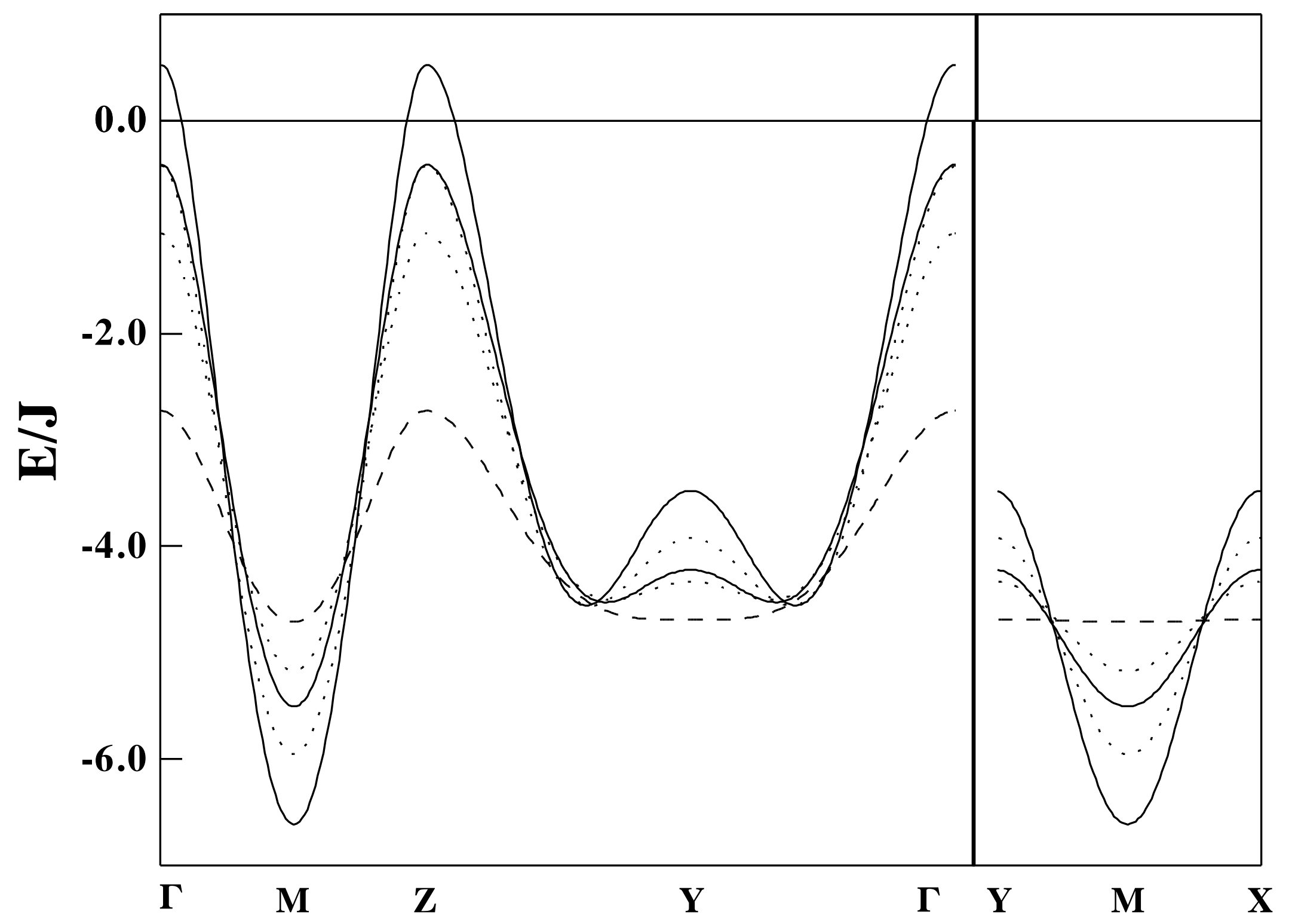


Fig. 4

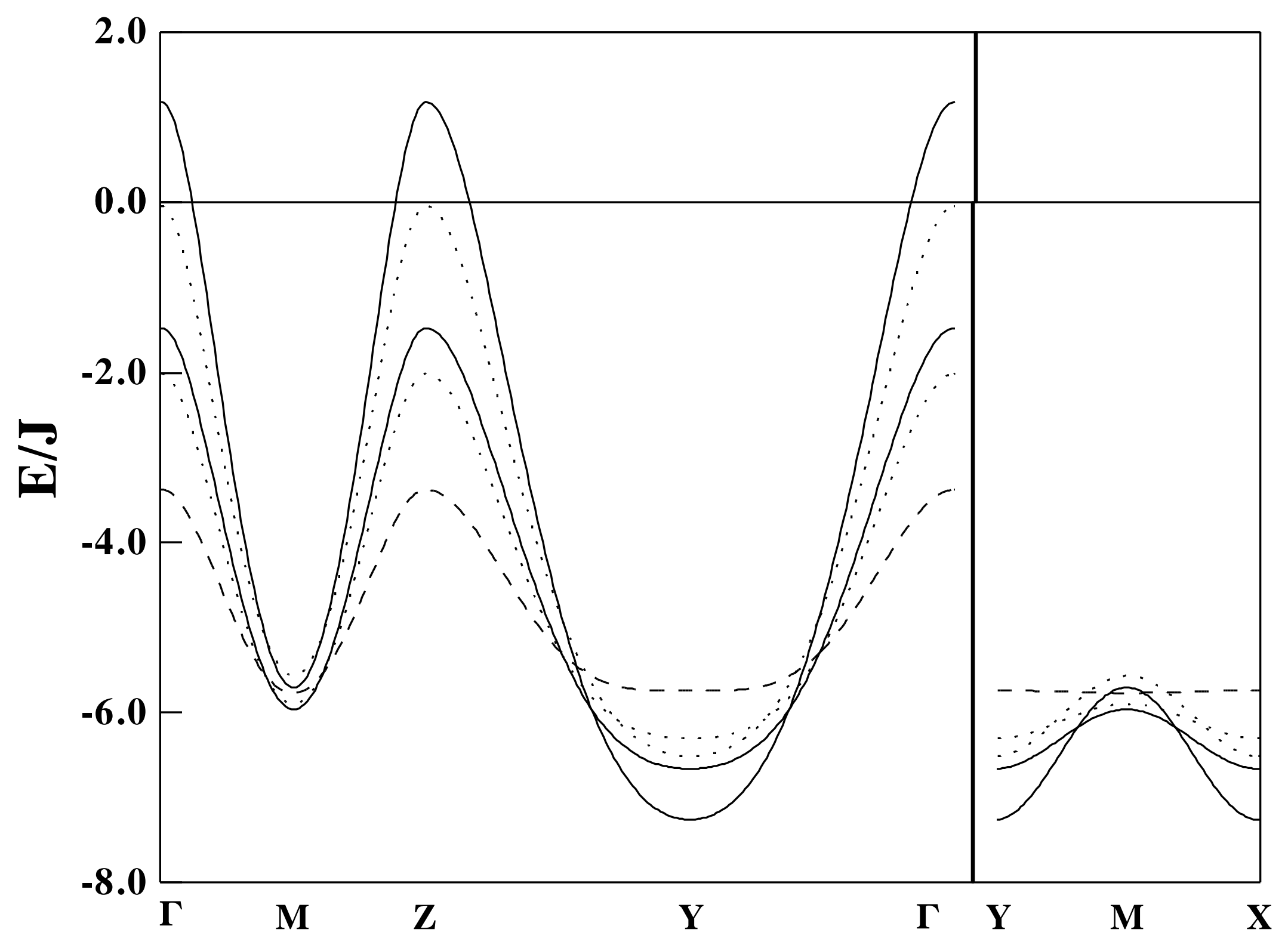


Fig. 5

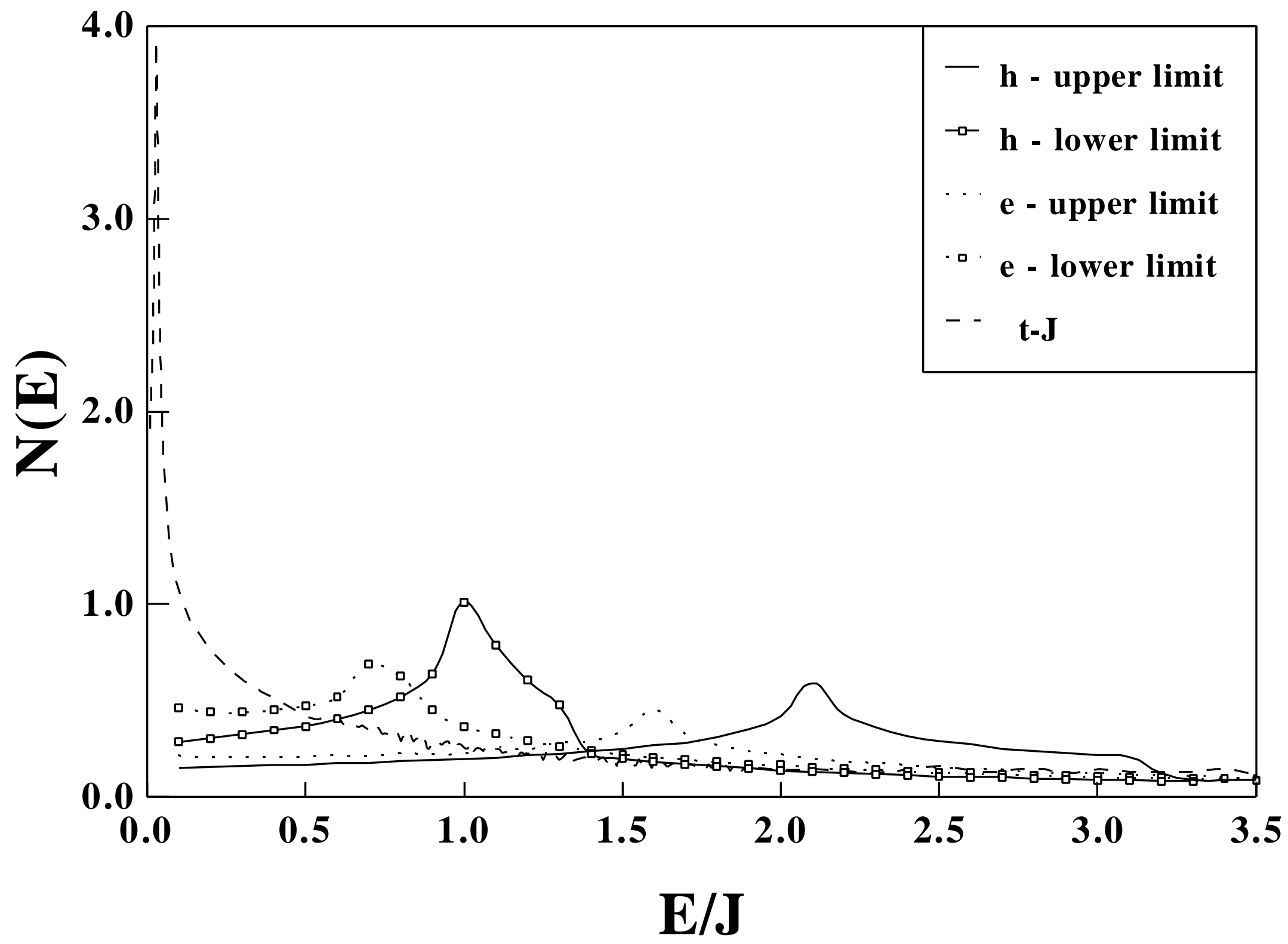


Fig. 6

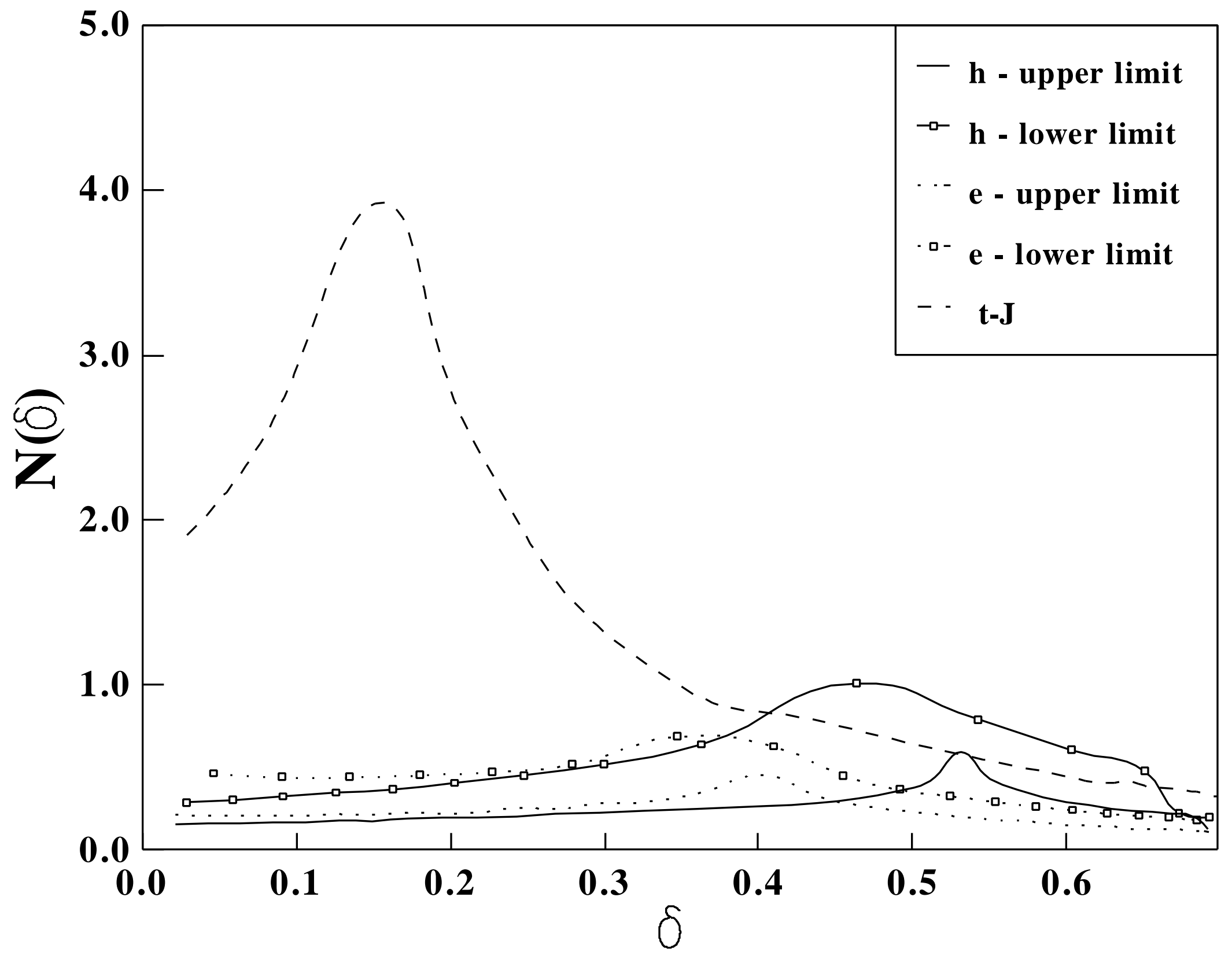

